\title{
Tramas tróficas de peces de arrecifes en la región noroccidental de Cuba. I. Contenido estomacal
}

\author{
Ivet Hernández, Consuelo Aguilar \& Gaspar González Sansón \\ Centro de Investigaciones Marinas, Universidad de La Habana, Calle 16 e/ 1ra y 3ra. No. 114, Miramar, Ciudad de \\ La Habana, CP 11300, Cuba; ivet@cim.uh.cu
}

Recibido 30-IV-2007. Corregido 29-III-2007. Aceptado 14-III-2007.

\begin{abstract}
Trophic webs of reef fishes in northwestern Cuba. I. Stomach contents. Studies on the reef fishes of Cuba are not rare, but most have two basic limitations: small sample sizes and exclusion of small species. Our study sampled more species and larger samples in the sublitoral region of Havana city $\left(23^{\circ} 7.587^{\prime} \mathrm{N}, 82^{\circ} 25.793^{\prime}\right.$ W), 2-18 m deep. We collected fish weekly from October 2004 through February 2006 with traps and harpoon. Overfishing has modified the fish communities. We used the relative importance index to describe the diets of carnivore and omnivore species, and a modification of the relative abundance method for the herbivores and sponge-eating species. The main food items are benthonic crustaceans (crabs, shrimp, copepods) and bony fish (mainly demersal species). Most species are eurifagous and thus, less affected by anthropic disturbance than specialist feeders. Rev. Biol. Trop. 56 (2): 541-555. Epub 2008 June 30.
\end{abstract}

Key words: stomach content, alimentation, reef fish, fish's diet, Cuba.

La alimentación es un factor determinante en la abundancia de las especies de peces y define la estructura de las comunidades limitando el número de especies e individuos que coexisten en una zona (Piet et al. 1998). Los peces tropicales tienen una gran variabilidad trófica, consumiendo más de una decena de taxa que pueden diferir en cada localidad y momento (Claro 1994). Randall (1967), hizo el análisis de la alimentación de la mayoría de los peces de arrecife del Caribe pero en muchos casos los tamaños de muestras son muy reducidos. En Cuba se han realizado varios estudios acerca de la alimentación en peces comerciales (Claro 1981a, 1981b, Sierra y Popova 1982, Aguilar et al. 1992), pero no en especies pequeñas. Pastor (2000), Faloh (2001) y Aguilar (2005), realizaron estudios de la alimentación de algunas de las especies chicas más abundantes u ornamentales pero utilizaron solamente el método frecuencial, lo cual fue una limitante al hacer algunos análisis cuantitativos. Son pocos los trabajos cuantitativos que se refieren a especies arrecifales de tallas pequeñas. Estas especies pueden tener gran importancia por su papel en la trama alimentaria del arrecife y por sus condiciones en el desarrollo de modelos biológicos debido a su elevada abundancia y fácil reproducción y mantenimiento en laboratorios. El presente trabajo tiene como objetivo determinar la composición de la dieta natural de la ictiofauna en el sublitoral rocoso de Ciudad de La Habana. Esta investigación constituye una contribución al conocimiento de la ecología trófica de los peces de arrecife.

\section{MATERIALES Y MÉTODOS}

El área de estudio es la porción del sublitoral de Ciudad de La Habana $\left(23^{\circ} 7.587^{\prime} \mathrm{N}\right.$, $82^{\circ} 25.793^{\prime} \mathrm{W}$ ), entre los 2 y $18 \mathrm{~m}$ de profundidad. En toda la zona las comunidades de peces han sido modificadas notablemente producto 
de la sobrepesca. Se muestreó semanalmente entre octubre del 2004 y febrero del 2006. Los peces fueron capturados mediante buceo autónomo empleando trampas de malla plástica tejida con una abertura cuadrada de $1 \mathrm{~mm}$ de trama para los individuos pequeños y arpón para los ejemplares de talla mediana y grande. Los especímenes se transportaron al laboratorio, donde se les determinó el peso húmedo en gramos con balanzas digitales tipo Denver de $1 \mathrm{mg}$ de precisión y la longitud de horquilla en $\mathrm{cm}$ con un ictiómetro. Los estómagos se conservaron en solución de formalina al $10 \%$ hasta su posterior análisis.

Cada entidad alimentaria encontrada se clasificó hasta el taxon más bajo posible empleando un microscopio estereoscópico. Las entidades alimentarias del contenido estomacal fueron contadas, medidas y pesadas, después de ser secadas con papel de filtro. Para describir las dietas de las especies se empleó el método del Índice de Importancia Relativa (IRI), definido como IRI $=(\% \mathrm{~N}+\% \mathrm{~W}) \mathrm{X} \%$ F por Pinkas et al. (1971) y el porcentaje de IRI como\% IRI $=100 \times \mathrm{IRI}_{\mathrm{i}} / \Sigma_{\mathrm{i}=1}^{\mathrm{n}} \mathrm{IRI}_{\mathrm{i}}$ (Morato et al. 2003). Para cada especie se realizaron curvas acumuladas de número de entidad alimentaria contra número de muestras tomadas para determinar si el tamaño de la muestra era suficiente donde la asíntota de la curva define el mínimo de estómagos requeridos. Para los herbívoros y comedores de esponjas se usó una modificación del método de abundancia relativa (Suárez et al. 1989), definiendo una escala adaptada del uno al tres con el objetivo de disminuir los sesgos. La escala quedó definida como: 1- Poco/Presente, 2- Normal y 3Abundante. Los análisis se realizaron usando el programa EXCEL 2003 para Windows. Ningún dato fue transformado ni estandarizado.

\section{RESULTADOS}

Fueron analizados 1561 estómagos en total de los cuales el $9.92 \%$ se encontraban vacíos. Se analizaron 22 especies de peces clasificadas como "carnívoras y omnívoras" y diez especies "herbívoras y comedoras de esponjas". Estos peces fueron los más abundantes, de más fácil captura y poco crípticos (Cuadro 1). Las tallas medias de todos los peces oscilaron entre los 4 y $40 \mathrm{~cm}$ de longitud.

\section{Caracterización general de la dieta}

Fueron identificados 165 componentes de la dieta de carnívoros y omnívoros, agrupados en 21 categorías teniendo en cuenta su importancia en la dieta de los peces (Cuadro 2). Cada categoría se considera como una entidad aparte a lo largo de la investigación. En general las entidades del infraorden Brachyura, resultaron ser uno de los grupos alimentarios principales dentro de la dieta de los peces (\% IRI=16.02). La familia mejor representada es Majidae (\% IRI=1.83). Holocentrus adscensionis, H. rufus y Serranus tigrinus, seguidos por Cephalopholis fulva son las especies que más se alimentan de los integrantes de este infraorden.

Los peces son la segunda entidad de mayor representatividad en las dietas (\% IRI=13.88). Las especies más ictiófagas son Caranx ruber, Lutjanus apodus, Bothus lunatus y Cephalopholis fulva. Dentro de los peces, la especie más consumida resultó ser Canthigaster rostrata, fundamentalmente por Myripristis jacobus (\% IRI=1.42).

Los huevos demersales se ingieren en gran número y frecuencia pero al presentar un peso menor, son menos representativos dentro de la dieta $(\%$ IRI=5.09). Las especies que más los ingieren son Stegastes partitus y Thalassoma bifasciatum. Los huevos pelágicos son consumido fundamentalmente por Chromis multilineata fundamentalmente (\% IRI=1.90). El aporte de las diferentes especies de copépodos no es despreciable (\% IRI=8.32). El orden Calanoida está bien representado en las dietas, especialmente los integrantes de la familia Candaciidae (\% IRI=8.15). Las especies Stegastes partitus, Thalassoma bifasciatum y Chromis multilineata son las que más copépodos ingieren. Capitellidae y Nereididae fueron las familias de mayor abundancia de poliquetos $\mathrm{y}$ son consumidas principalmente por las especies pertenecientes al género Chaetodon (\% IRI=5.58). 
TABLA 1

Especies seleccionadas para la investigación. La clasificación se realizó siguiendo los criterios de Eschmeyer (1998), para los nombres cientificos y de Guitar (1978), para los nombres comunes

TABLE 1

Research selected species. Cientific names clasification was according to Eschmeyer (1998).

Vulgar names classification was according to Guitar (1978)

Especies

Acanthostracion polygonius (Poey, 1876)

Acanthurus bahianus (Walbaum, 1792)

Acanthurus chirurgus (Bloch, 1787)

Acanthurus coeruleus (Bloch y Schneider,1801)

Bothus lunatus (Linnaeus, 1758)

Cantherhines pullus (Ranzani, 1842)

Caranx ruber (Bloch, 1793)

Cephalopholis fulva (Linnaeus, 1758)

Chaetodon capistratus (Linnaeus, 1758)

Chaetodon ocellatus (Bloch, 1787)

Chaetodon striatus (Linnaeus, 1758)

Chromis multilineata (Guichenot, 1853)

Diodon holocanthus (Linnaeus, 1758)

Equetus acuminatus (Bloch y Schneider,1801)

Haemulon plumieri (Lacepéde, 1801)

Haemulon sciurus (Shaw, 1803)

Halichoeres garnoti (Valenciennes, 1839)

Holacanthus ciliaris (Linnaeus, 1758)

Holocentrus adscensionis (Osbeck, 1765)

Neoniphon marianus (Cuvier, 1829)

Holocentrus rufus (Walbaum, 1792)

Lutjanus apodus (Walbaum, 1792)

Lutjanus synagris (Linnaeus, 1758)

Myripristis jacobus (Cuvier, 1829)

Pomacanthus paru (Bloch, 1787)

Rypticus saponaceus (Bloch y Schneider,1801)

Sparisoma aurofrenatum (Valenciennes, 1840)

Sparisoma chrysopterum (Bloch y Schneider,1801)

Sparisoma viride (Bonnaterre, 1788)

Serranus tigrinus (Bloch, 1790)

Stegastes partitus (Poey, 1868)

Thalassoma bifasciatum (Bloch, 1791)
Nombre vulgar

Código

Torito hexagonal

Lacpol

Barbero

Abah

Barbero

Achi

Barbero azul

Acoe

Lenguado

Botlun

Lija

Canpul

Civí carbonero

Cararub

Guatívere

Cepful

Parche ocelado

Chacap

Parche amarillo

Chaocel

Parche rayado

Chastri

Cromis prieto

Chromul

Pez erizo

Diohol

Vaqueta rayada

Equacum

Ronco arará

Haeplum

Ronco amarillo

Haesciu

Doncella de cara amarilla

Halgar

Angelote reina

Holcil

Carajuelo de ascensión

Holasd

Carajuelo mariano

Neomar

Carajuelo rufo

Holruf

Cají

Lutapo

Biajaiba

Lutsyn

Candil barreado

Myrjac

Angelote francés

Pompar

Jaboncillo

Rypsap

Loro

Spauro

Loro

Spachry

Loro

Spavir

Serrano tigre

Sertig

Chopita bicolor

Stepar

Doncella cara de cotorra
Thabif 


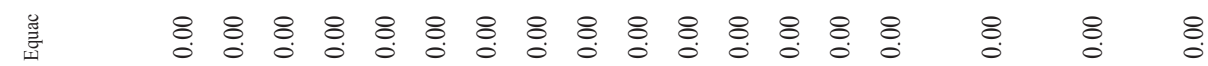

章 \& \& \& \& \& \&

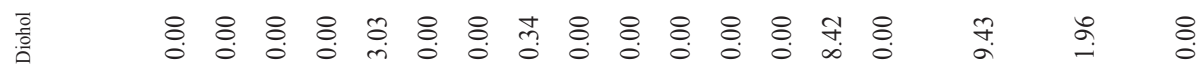

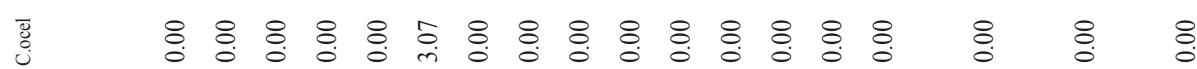

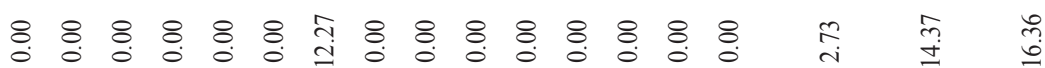

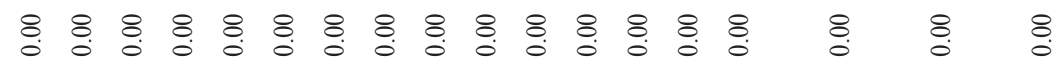

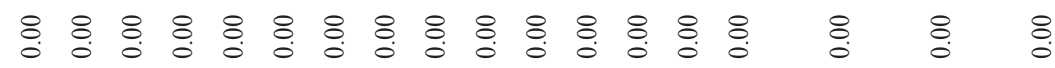

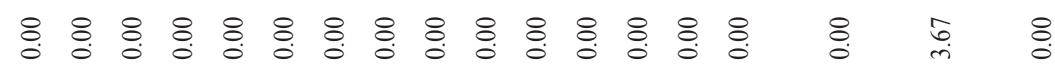

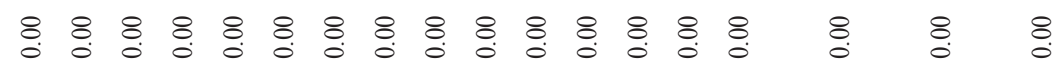

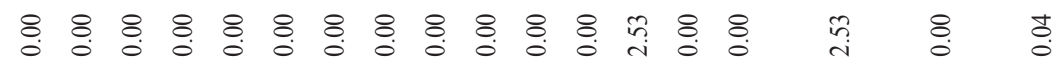

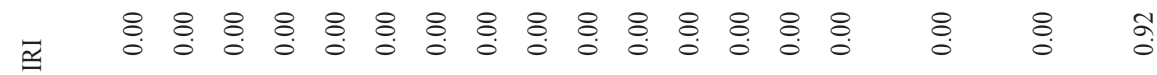

\& $8.80 .0 \%$

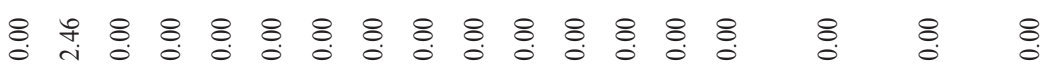

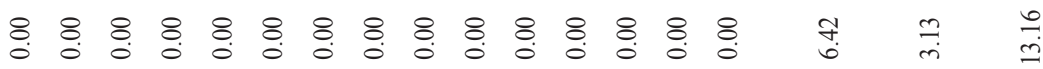

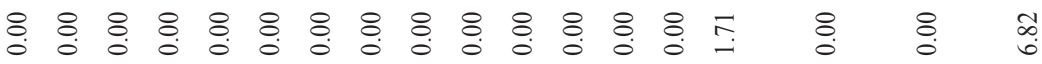

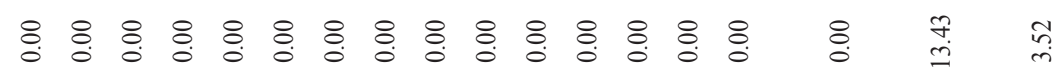

ฐ

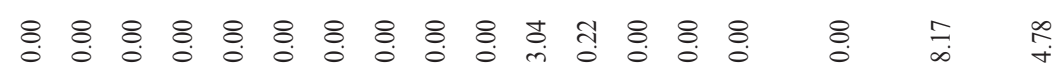

= $8.8 \%$

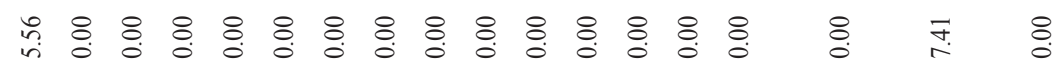

గి

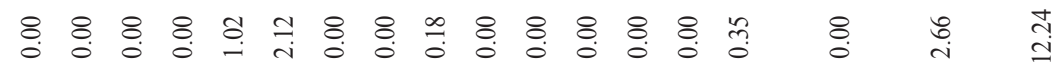

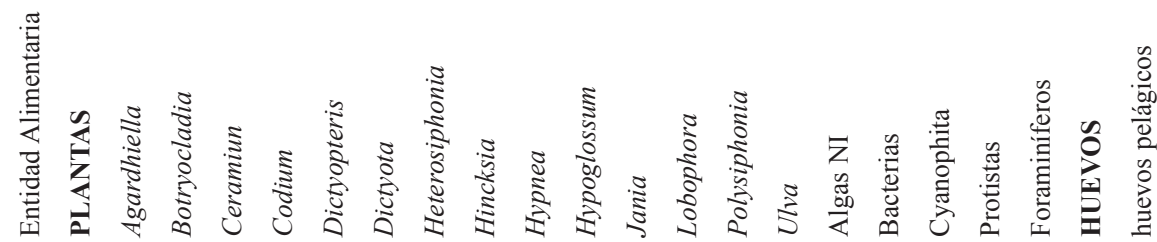
藏一 
親

章

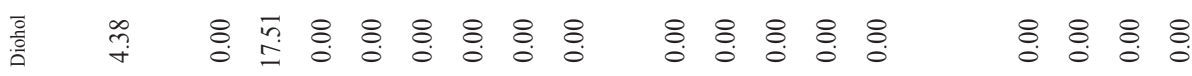

ग

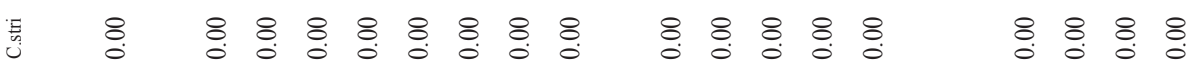

屋

言

衰

望

营

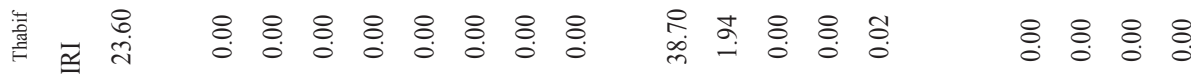

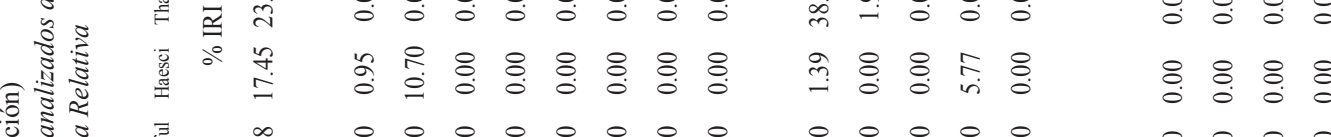

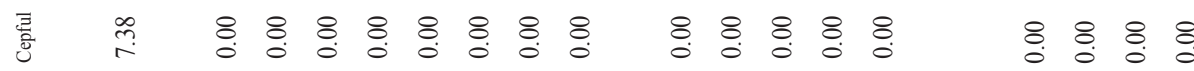

衰

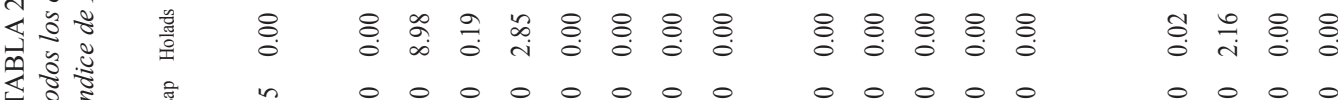

变

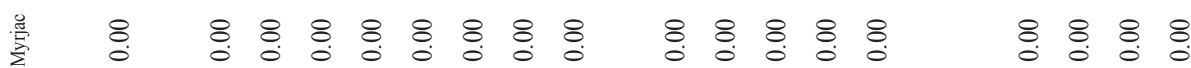

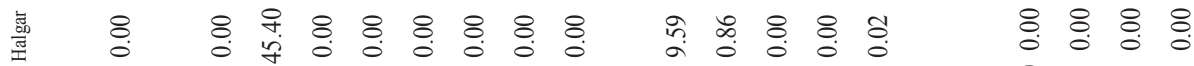

留

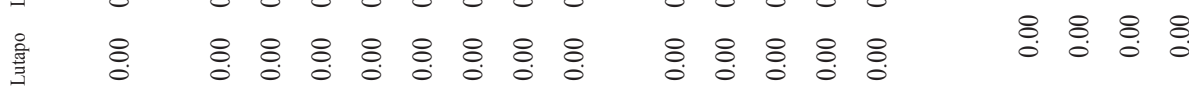

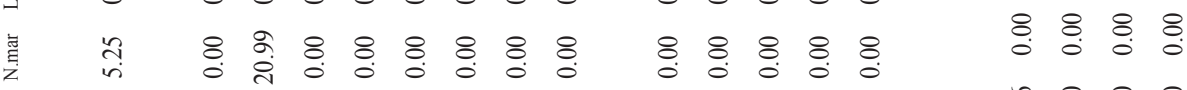

言 भ

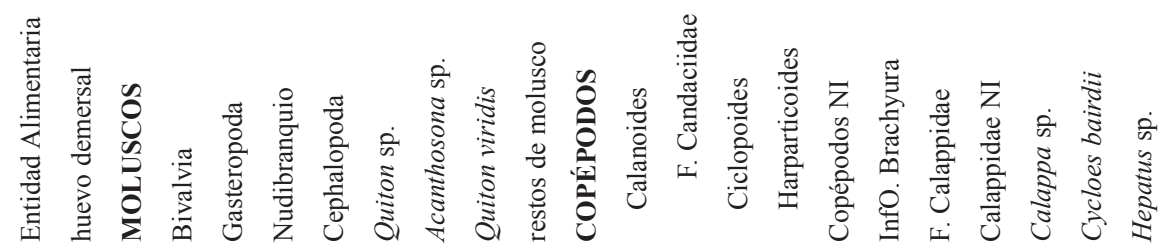

葽的ナ 
意 $8: 8 \%$

竞

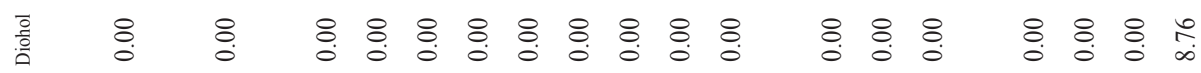

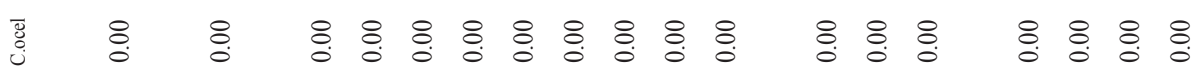

镸

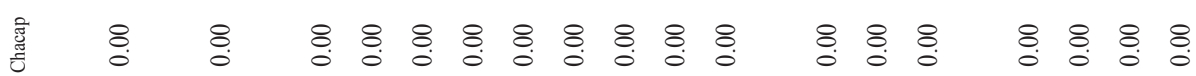

言

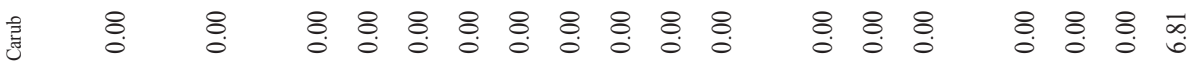

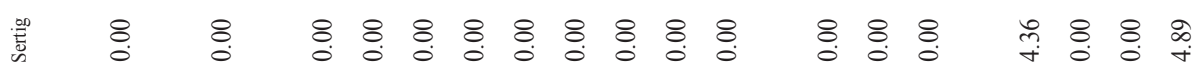

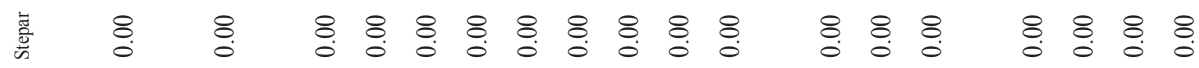

衰

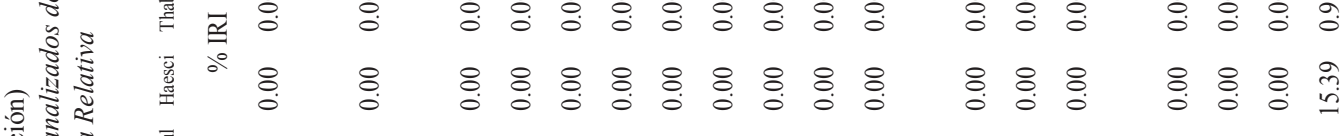

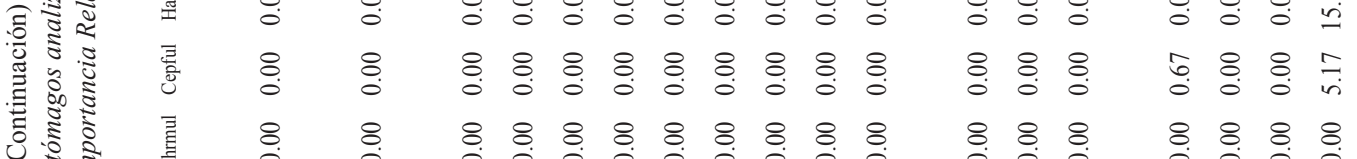

武 8

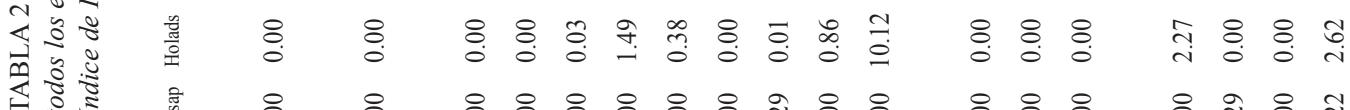

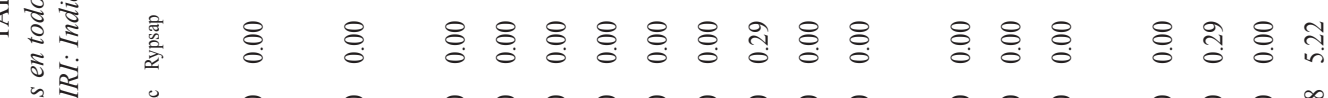

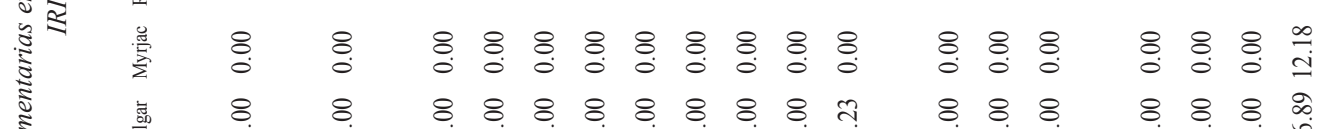

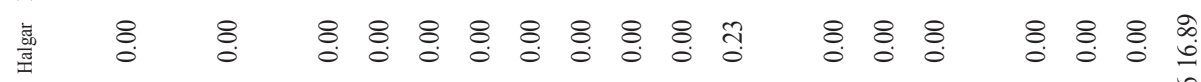

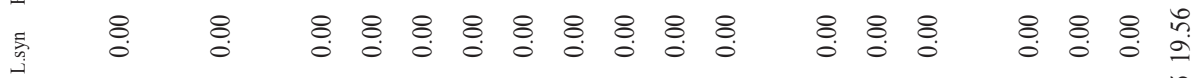

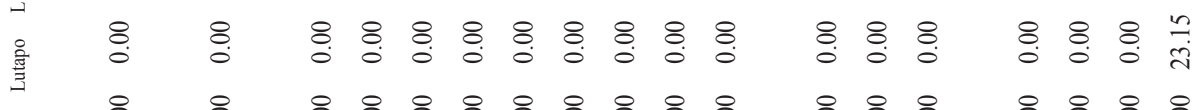

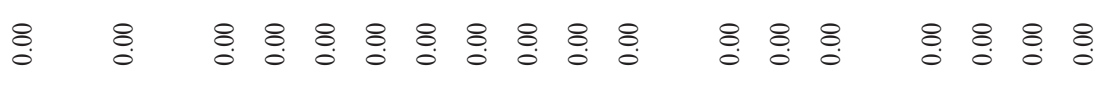

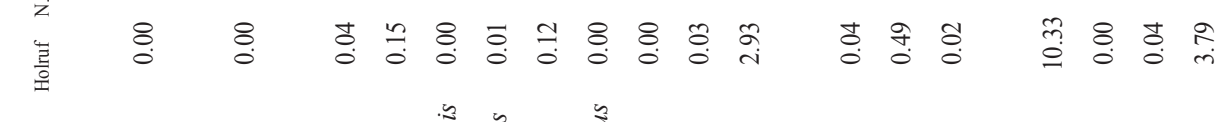

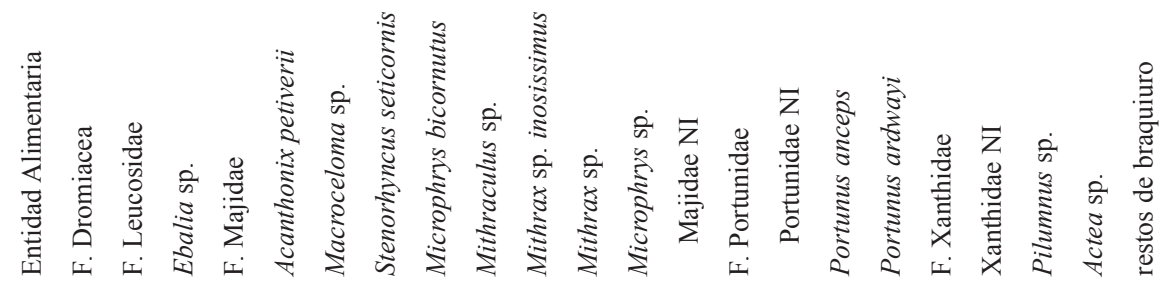

芒 $\infty$

$\therefore \quad=$ 
兹 \&

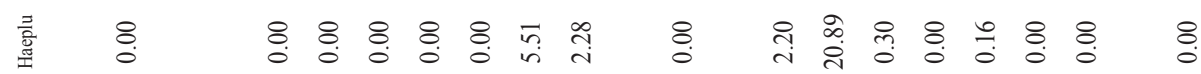

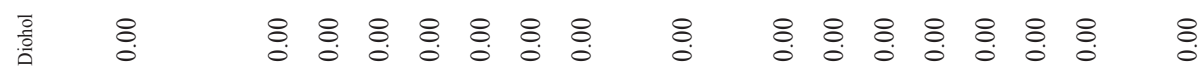

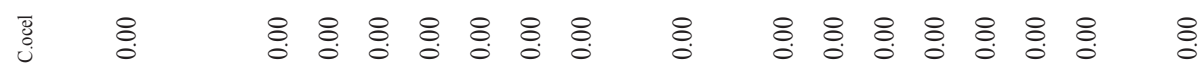

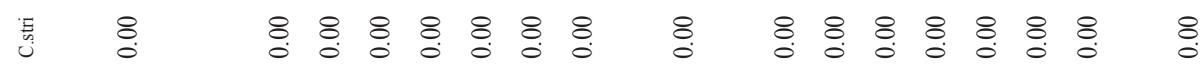

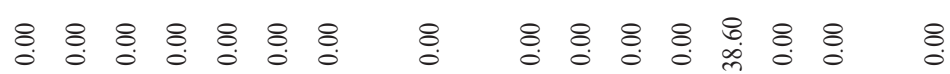

:

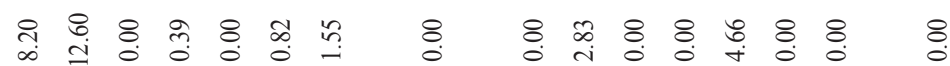

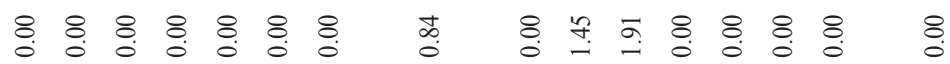

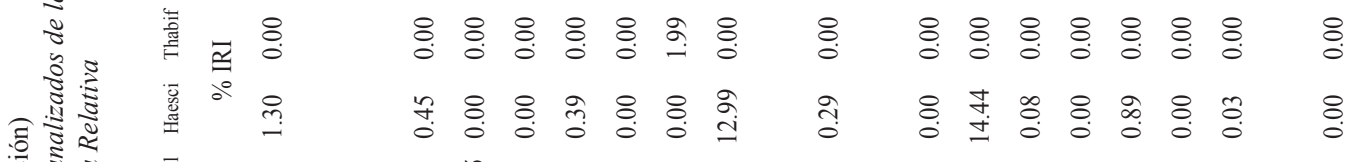

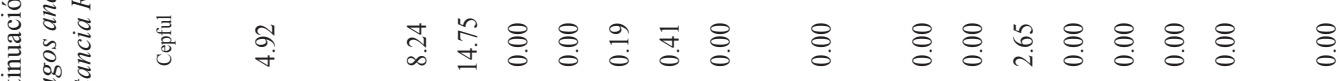

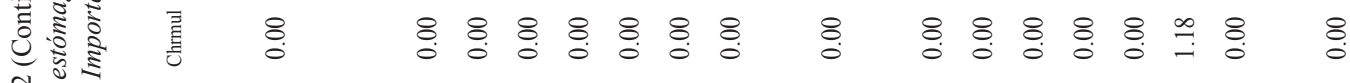

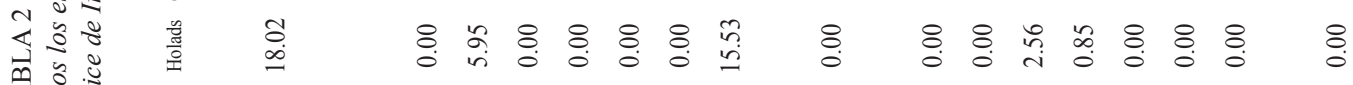

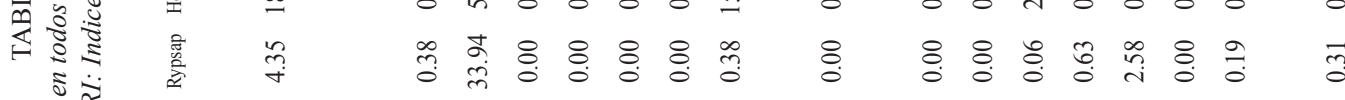

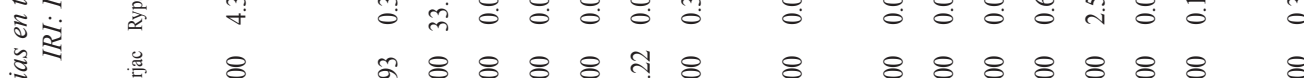

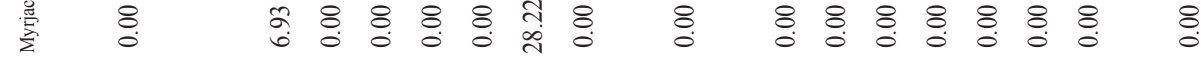

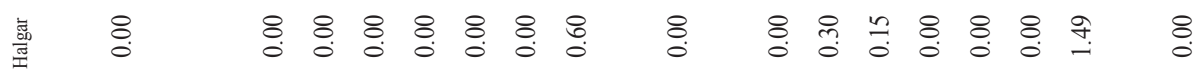

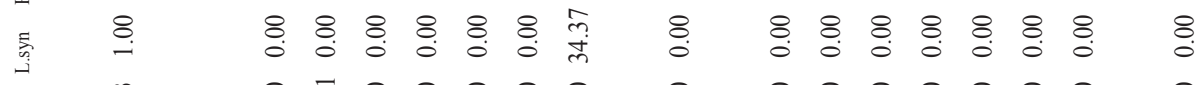

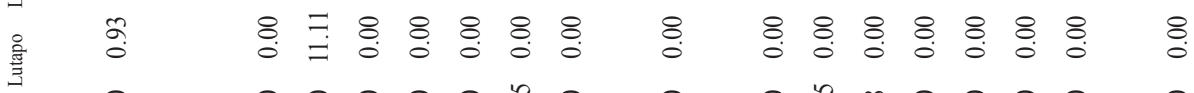

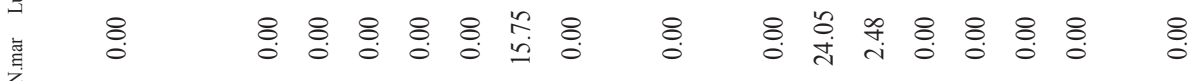

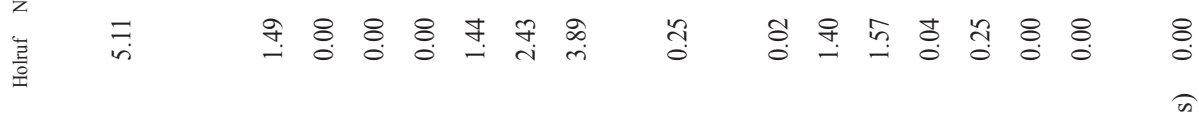

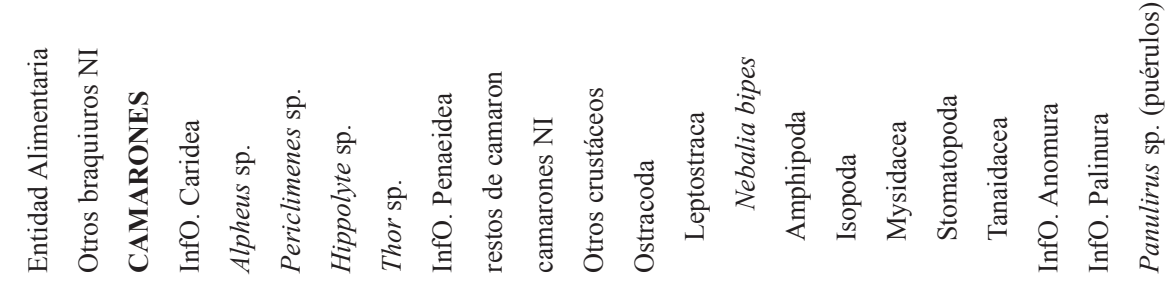

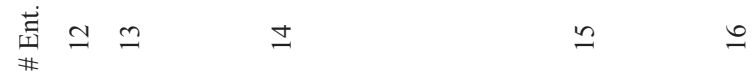




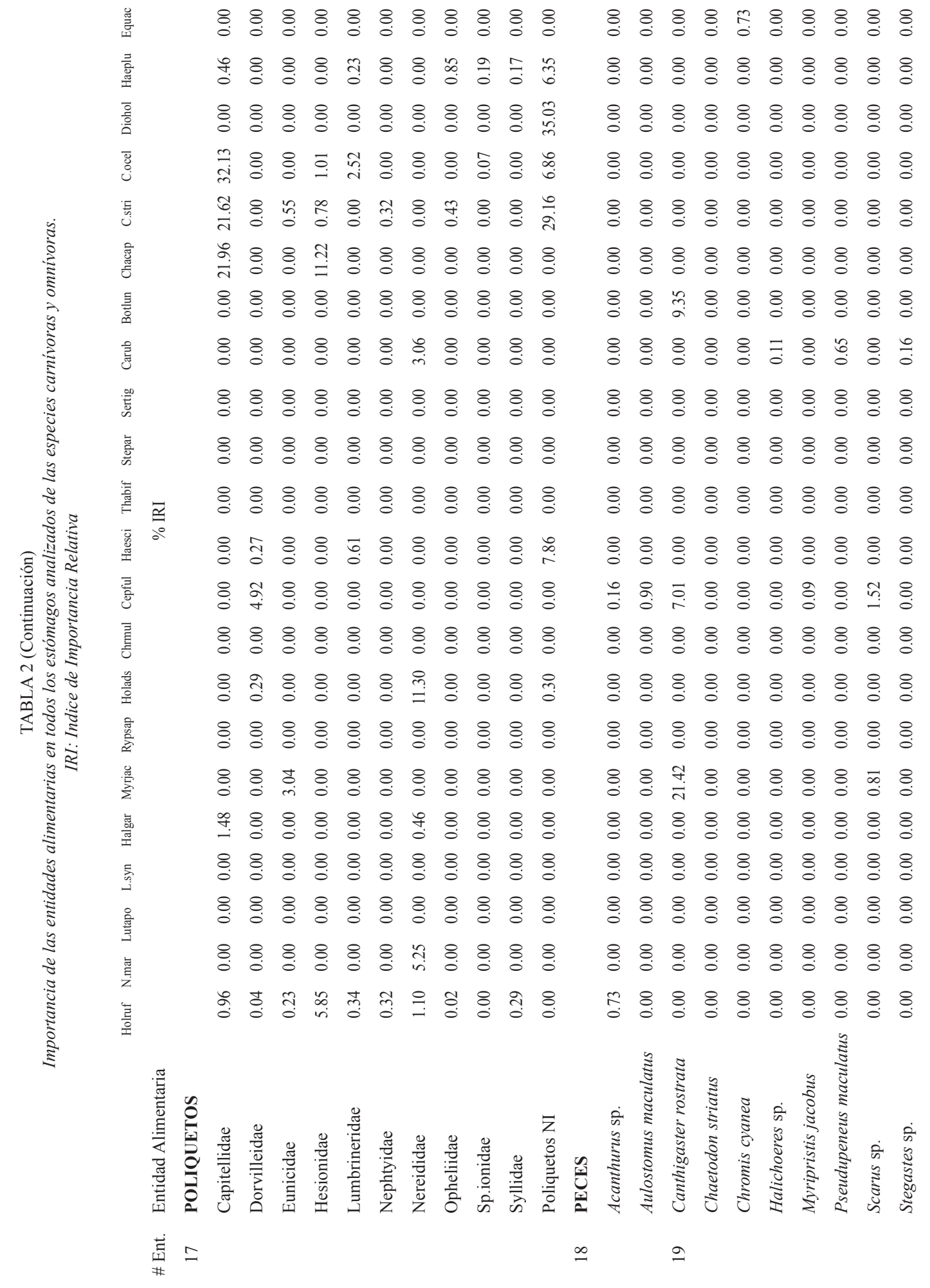


善 $8 \% 8 \%$

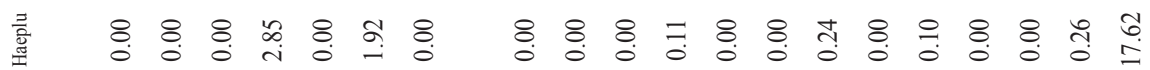

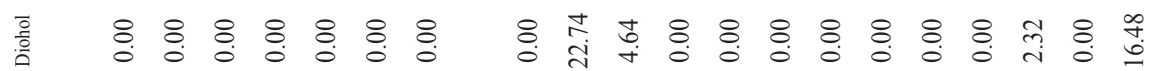
悉 8 镸

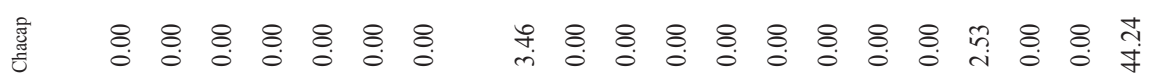

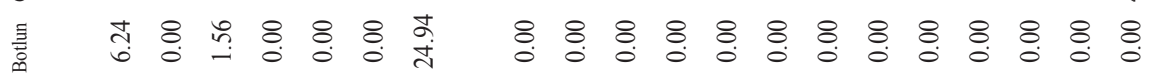
急 㷰 衰 琗 遑 衰 $8080.0 \%$

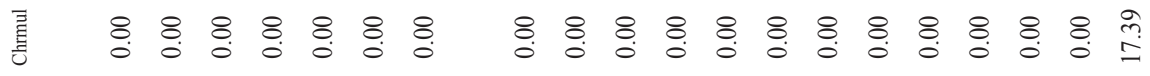

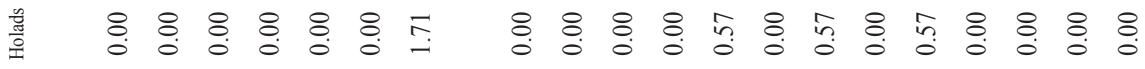

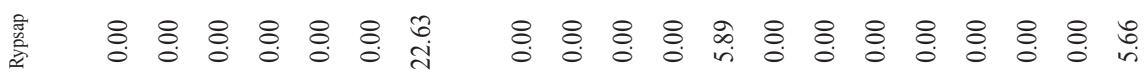
इ 皇

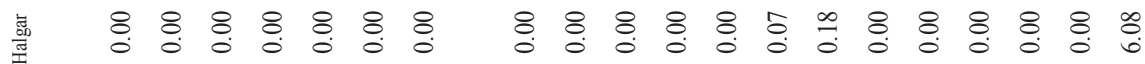

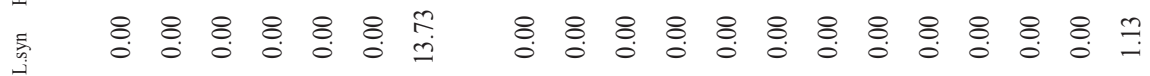

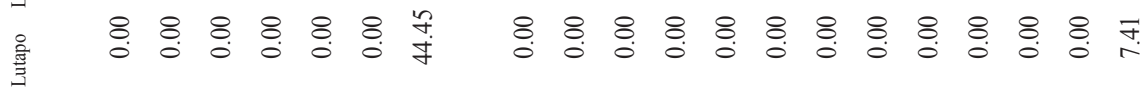
:

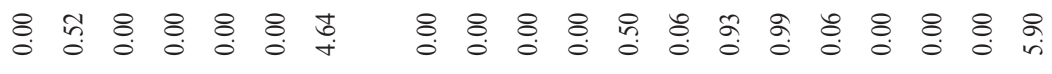

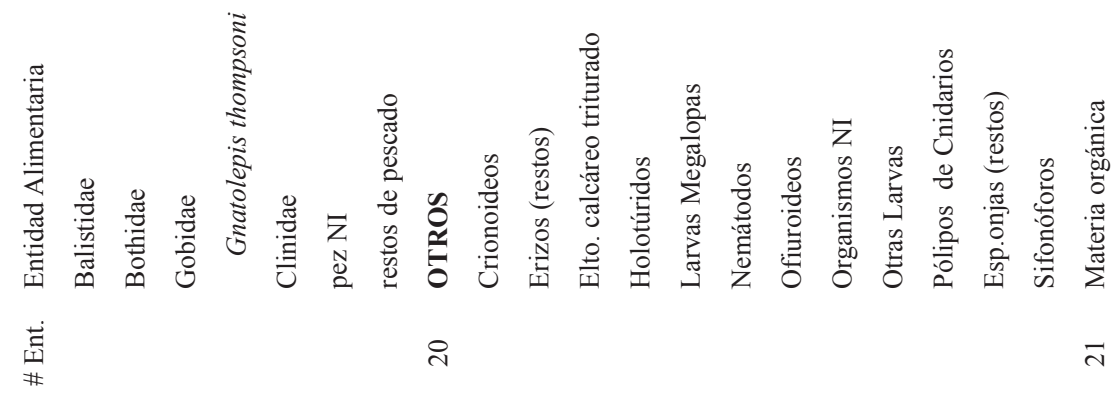


Las algas del género Heterosiphonia son las más ingeridas por los peces $(15 \%)$, fundamentalmente por las especies Chaetodon striatus y Neoniphon marianus. Los géneros Agardhiella (8.8\%) y Jania (11.6\%), segundos en importancia, son consumidos fundamentalmente por las especies Lutjanus apodus, L. synagris y Neoniphon marianus, respectivamente. Codium sp., es la menos representada en las dietas. Las especies que más moluscos consumen son Halichoeres garnoti, Neoniphon marianus y Diodon holocanthus, especialmente de la clase Gasteropoda (\% IRI=3.18). Finalmente, las entidades alimentarias más representativas de la categoría "otros" fueron la materia orgánica (compuesta por restos de tejidos) (\% IRI=14.84), consumida por todas las especies y los crinoideos juveniles. Estos últimos forman la parte principal de la dieta de los parches.

De todas las especies de herbívoros y consumidores de esponjas estudiadas, el $45.5 \%$ consume algas como alimento principal, representando estas el $71.55 \%$ de su dieta. El análisis de los tractos digestivos de todos los peces permitió confeccionar la lista general del contenido estomacal. Se clasificaron 59 entidades, de ellas, 50 fueron macroalgas, identificadas hasta el nivel de género (Cuadro 3). Las especies pertenecientes al género Acanthurus fueron las de mayor consumo de algas desde el punto de vista volumétrico. Para los herbívoros, se define a la categoría "otros" como: elementos calcáreos triturados, arena y piedras.

\section{DISCUSIÓN}

La composición de la dieta de los peces examinados muestra que las principales entidades alimentarias fueron los crustáceos bentónicos (cangrejos, camarones, copépodos) y peces óseos, mayoritariamente demersales. Resultados similares han sido descritos por otros autores (Vega-Cendejas et al. 1994, Piet et al. 1998, Sierra et al. 2001, Arias-González et al. 2004).

Frecuentemente se encontraron fragmentos calcáreos de algas, foraminíferos, espículas de esponja, gastrópodos, erizos, ofiuroideos, piedras, arena y cianofitas en el estómago de los carnívoros. Es muy probable que hallan sido ingeridas accidentalmente en el intento de atrapar a su verdadera presa. La mayoría de este material no pudo ser identificado por lo deteriorado que se encontraba. Estos resultados concuerdan con Randall (1967), quien plantea que en ocasiones, el material estomacal de las presas puede ser atribuido erróneamente a los depredadores, como la arena ingerida por poliquetos.

Los representantes de la familia Lutjanidae son mayormente piscívoros, aunque consumen también gran cantidad de crustáceos y organismos bentónicos. Fueron hallados camarones, especialmente pertenecientes al género Periclimenes, en los estómagos de Lutjanus synagris y Lutjanus apodus, lo que está en concordancia con otros autores (Edgar y Shaw 1995, Rooker 1995, Sierra et al. 2001). Esto resulta contrario a lo registrado por Cocheret et al. (2003), quien reportó la ausencia de estos crustáceos en todos los ejemplares de Lutjanus spp. que estudió.

Los roncadores (Haemulidae) se alimentan de una gran variedad de invertebrados. La composición de la dieta encontrada en la presente investigación para esta familia, concuerda con la reportada por diferentes autores (Randall 1967, Claro 1983, Vega-Cendejas et al. 1994, Sierra et al. 2001).

Los integrantes de la familia Serranidae se alimentan de una amplia variedad de peces e invertebrados de diversos tamaños (Sadovy y Eklund 1999). Están entre los carnívoros más importantes de los arrecifes de coral. Los resultados en esta investigación sobre la especie Cephalopholis fulva, concuerdan con los reportados por varios autores (Carter et al. 1994, Sullivan y De Garine-Wichatitsky 1994, García-Arteaga et al. 1999). Las especies depredadoras del área de estudio, presentan tallas pequeñas, debido con probabilidad, al efecto de la sobrepesca. El control que realizan lo hacen a su vez, sobre especies de tallas chicas. Esto podría constituir un control realizado por estas especies sobre las presas en etapas 
TABLA 3

Lista de las entidades alimentarias encontradas en tractos digestivos de herbivoros y comedores de esponjas

$(\% \mathrm{AR})$

\begin{tabular}{|c|c|c|c|c|c|c|c|c|c|c|}
\hline Entidades & Spachry & Spauro & Spavir & Lacpol & Acabah & Acacoe & Acachi & Holcil & Canpul & Pompar \\
\hline Acanthophora & & & & & & 0.22 & & & & \\
\hline Acetabularia & & & & & & & 0.09 & & & \\
\hline Agardhiella & 1.65 & 1.20 & 1.03 & 1.40 & 6.00 & 2.60 & 3.52 & 3.47 & & 5.47 \\
\hline Aglaothamnion & & 1.61 & 1.03 & & 1.32 & 0.43 & 1.25 & & & \\
\hline Alga NI & 1.65 & 0.81 & & & 0.44 & 2.17 & 0.47 & 0.99 & & 5.01 \\
\hline Alga verde NI & & & & & 0.29 & & 1.20 & & & 1.37 \\
\hline Amphiroa & & 0.40 & & & & 1.45 & 0.30 & & & \\
\hline Anadyomene & & & 22.64 & & 0.73 & 0.29 & 1.20 & & & \\
\hline Bostrychia & & & & & 0.44 & & & & & \\
\hline Botryocladia & & & & & & 0.36 & & & & 3.64 \\
\hline Bryothamnion & & & & & & 0.72 & & & & \\
\hline Caloglossa & & & & & & 0.14 & 0.26 & & & \\
\hline Caulerpa & & & & & & & 0.04 & & & 12.75 \\
\hline Centroceras & & 8.46 & 7.20 & & 2.49 & 2.03 & 1.38 & & & \\
\hline Ceramiun & 13.18 & 3.22 & & & 4.83 & 1.59 & 3.31 & & & \\
\hline Champia & & & & & 0.15 & 0.29 & 0.04 & & & \\
\hline Chondria & & & & & & 0.51 & 0.13 & & & \\
\hline Cladophora & & & 1.54 & & & & 1.89 & & & \\
\hline Cladophoropsis & & & & & & 0.14 & 0.17 & & & \\
\hline Codium & & & & 4.20 & & 1.81 & 0.34 & & & 8.20 \\
\hline Dasycladus & & & & & & 1.74 & & & & \\
\hline Dictyopteris & 1.65 & 0.40 & & & 0.44 & 1.23 & 1.89 & 12.87 & & 3.64 \\
\hline Dictyota & & & 10.81 & & 5.12 & 9.99 & 4.00 & & 3.41 & 8.20 \\
\hline Enteromorpha & & 4.03 & & & 0.88 & & 1.80 & 3.96 & & \\
\hline Galaxaura & & 10.07 & 6.69 & & 4.83 & 7.81 & 2.28 & & & \\
\hline Gelidiella & & & & & 3.81 & 5.14 & 1.03 & & & 3.64 \\
\hline Gelidiopsis & & & & & 2.05 & 2.68 & 3.09 & 4.95 & & 3.64 \\
\hline Gelidium & 6.59 & 2.42 & 1.54 & & 0.88 & 1.37 & 0.90 & & & \\
\hline Gracilaria & & & & & 0.88 & 1.81 & 0.13 & 3.96 & & 0.46 \\
\hline Griffithsia & & & & & 0.29 & & & & & \\
\hline Halimeda & 11.53 & 2.82 & & 7.00 & 6.30 & 6.44 & 6.79 & 9.90 & 18.21 & 8.65 \\
\hline Haliptilon & & & & & 0.29 & & 0.04 & & & \\
\hline Heterosiphonia & 6.59 & 1.61 & & & 6.73 & 4.56 & 6.40 & & & \\
\hline Hincksia & & 0.81 & & & 0.73 & & 0.34 & & & \\
\hline
\end{tabular}


TABLA 3 (Continued)

Lista de las entidades alimentarias encontradas en tractos digestivos de herbivoros y comedores de esponjas

$$
(\% \mathrm{AR})
$$

\begin{tabular}{|c|c|c|c|c|c|c|c|c|c|c|}
\hline Entidades & Spachry & Spauro & Spavir & Lacpol & Acabah & Acacoe & Acachi & Holcil & Canpul & Pompar \\
\hline Hydropuntia & & & & & & 0.36 & & & & \\
\hline Hypnea & & 1.61 & & & 2.05 & 6.80 & 3.52 & 1.98 & & \\
\hline Hypoglossum & & & & & 0.29 & 2.24 & 1.20 & & & 1.37 \\
\hline Jania & & 0.81 & 1.54 & & 8.49 & 6.80 & 7.78 & & 2.28 & \\
\hline Laurencia & & & & & & 0.87 & & & & \\
\hline Lobophora & 4.94 & 1.61 & & & 1.46 & 0.14 & 0.52 & & & 4.10 \\
\hline Microdictyon & & & & & & 0.07 & 0.86 & & & \\
\hline Polysiphonia & & 5.64 & & & 0.88 & & 1.50 & & & 2.74 \\
\hline Pterocladiella & & & & & & 0.51 & & & & \\
\hline Rhodomelaceae & & & & & & 0.72 & & & & \\
\hline Scinaia & & & & & & 0.43 & & & & \\
\hline Solieria & & & & & 1.90 & 1.45 & 1.16 & & & \\
\hline Sphacelaria & & & & & 0.44 & & & & & \\
\hline Udotea & & & & & 0.15 & 0.14 & 0.04 & & & 0.91 \\
\hline Ulva & & 4.43 & 6.18 & & 4.25 & 0.36 & 1.85 & & & \\
\hline Ventricaria & & & & & & 0.36 & & & & \\
\hline Cianofitas & 13.18 & 6.04 & & 4.20 & 3.51 & 7.53 & 7.13 & 3.96 & 1.14 & 3.64 \\
\hline Copépodos & & & 0.51 & & & 0.29 & 0.09 & & & \\
\hline Esponjas & 4.94 & 1.21 & & 20.80 & 0.29 & & 0.09 & 46.53 & 69.12 & 17.56 \\
\hline Huevos & & 2.42 & 8.75 & 4.20 & 0.29 & 0.14 & 2.75 & 1.49 & & \\
\hline Materia orgánica & 32.15 & 17.29 & 8.35 & 22.67 & 1.17 & 1.52 & 3.22 & 5.45 & 3.28 & 4.10 \\
\hline Moluscos & & 4.83 & & & 1.90 & 0.07 & 2.45 & & & \\
\hline Otros & 1.94 & 16.28 & 20.13 & 5.88 & 22.69 & 11.36 & 20.84 & & 2.56 & 0.91 \\
\hline Otros crustáceos & & & & 5.62 & & 0.29 & 0.13 & & & \\
\hline Poliquetos & & & 2.06 & 42.02 & 0.29 & & 0.56 & 0.50 & & \\
\hline
\end{tabular}

$\mathrm{AR}=\mathrm{Abundancia}$ Relativa.

tempranas de su desarrollo. Los peces adultos podrían, tal vez, constituir presa de depredadores mayores pero éstos no abundan en el área.

La dieta hallada durante este trabajo para los individuos de la especie Caranx ruber, está en concordancia con la reportada por Randall (1967) y por Sierra y Popova (1982). Una de las presas es la especie Stegastes partitus, la cual conforma el $42 \%$ de las comunidades de peces de la zona de estudio (HernándezHernández et al. 2006). Teniendo en cuenta la baja abundancia de Caranx ruber en el área y su carácter transitorio en los arrecifes, no se estima que esta especie ejerza un control regulador importante sobre las poblaciones de Stegastes partitus. 
En Halichoeres garnoti se encontró como alimento principal los gastrópodos, lo que concuerda con estudios de Cervigón (1993) y Sierra et al. (2001). Además consume algas, por lo que Beebe y Tee-Van (1928) lo consideraron como omnívoro. Cervigón (1993) y Aguilar (2005), coinciden con los resultados mediante este estudio para la especie Thalassoma bifasciatum.

Los hábitos alimentarios de los individuos de la familia Pomacentridae resultaron ser muy similares a los encontrados en otras investigaciones (Hixon y Beets 1993, Booth y Hixon 1999, Aguilar 2005). A diferencia de Thalassoma bifasciatum, que depreda únicamente huevos demersales de otras especies, el consumo de huevos por Stegastes partitus, está dirigido hacia algunos huevos de su propia camada con el fin de permitir que el agua con oxígeno circule mejor entre los restantes y aumentar su supervivencia (Payne et al. 2004).

La información brindada por Randall (1967) y Bouchon-Navarro (1986), sobre la composición de la dieta de los parches (Chaetodon spp.), es consistente con lo encontrado en este trabajo. Teniendo en cuenta la poca abundancia de individuos de crinoideos de talla grande en el área de estudio, es posible considerar que estos peces ejercen un control regulador de la población. La baja diversidad de entidades alimentarias encontradas en el contenido estomacal de estas especies, en comparación con otros peces, sugiere que los integrantes de la familia Chaetodontidae pueden ser considerados como estenófagos.

La composición de la dieta de los integrantes de la familia Holocentridae, coincide notablemente con lo consignado por Randall (1967) y Arias-González et al. (2004). Ambos autores han reportado la aparición de algas en el contenido estomacal de estos peces junto con sus presas. Esto se verifica especialmente en Neoniphon marianus. El hecho de que la especie Myripristis jacobus se alimente de numerosos peces en estadio juvenil, podría constituir un control sobre sus presas en etapas tempranas.

La dieta de Diodon holocanthus resultó muy variada. Se ha reportado oportunismo trófico para las especies de la familia Diodontidae, producto del elevado grado de eurifagia de estos peces y que puede estar atribuido al gran número de especies de la fauna del arrecife y al diverso y abundante suministro de alimento (Sierra et al. 2001).

Se sugiere que para la mayoría de las especies estudiadas, la estrategia trófica dominante es la eurifagia. Aunque puedan encontrarse muchas especializaciones en la dieta de algunas especies, estas adaptaciones pueden variar en dependencia del alimento local hallado. Los resultados en esta investigación concuerdan con esa información.

Vega-Cendejas etal.(1994)y Salamunovich y Ridenhour (1990), concuerdan con que la mayoría de las especies incluidas en el presente estudio, son generalistas debido al amplio espectro de sus dietas. No obstante, opinan que existe suficiente segregación de recursos entre las distintas especies, por lo que se disminuye su competencia y esto les permite coexistir. Macpherson (1981), reportó que los generalistas pueden explotar mejor las diversas fuentes de alimento y son capaces de sobrevivir en ambientes fluctuantes. Sin embargo, las especies estenófagas ocupan nichos tróficos estrechos y son inmediatamente impactados por las perturbaciones o el estrés en la comunidad.

\section{RESUMEN}

Se analizó el contenido estomacal de los peces arrecifales más abundantes de una zona del sublitoral rocoso de Ciudad de La Habana, desde octubre del 2004 hasta febrero del 2006. Para describir las dietas de las especies de peces carnívoras y omnívoras se empleó el índice de importancia relativa. Para los herbívoros y consumidores de esponjas se usó una modificación del método de abundancia relativa. La mayoría de las especies estudiadas son eurífagas y consumen principalmente otros peces y crustáceos bénticos.

Palabras clave: contenido estomacal, alimentación, peces de arrecife, dieta de peces, Cuba.

\section{REFERENCIAS}

Aguilar Betancourt, C. 2005. La ictiofauna costera de Ciudad de La Habana: Efectos acumulativos de agentes estresantes múltiples en varios niveles de 
organización biológica. Tesis de Doctorado, Centro de Investigaciones Marinas, Universidad de La Habana, La Habana, Cuba.

Aguilar Betancourt, C., G. González-Sansón \& T. Veledo Alemán. 1992. Alimentación natural de jóvenes de rabirrubia (Ocyurus crysurus) (Blosh), en una de las plataformas occidentales de Cuba. Rev. Invest. Mar. 1: 35-39.

Arias-González, J., E.R. Galzin \& M. Harmelin-Vivien. 2004. Spatial, ontogenetic and temporal variation in the feeding habits of the squirrelfish Sargocentron microstoma on reefs in Moorea, French Polynesia. Bull. Mar. Sci. 75: 473-480.

Beebe, W. \& J. Tee-Van. 1928. The fish of Port-au-Prince Bay, Haití with a summary of the known species of marine fish of the island of Haití and Santo Domingo. Zoologica 10: 1-279.

Booth, D.J. \& M.A. Hixon. 1999. Food ration and condition affect early survival of the coral reef damselfish, Stegastes partitus. Oecologia 121: 364-368.

Bouchon-Navaro, Y. 1986. Partitioning of food and space resources by chaetodontid fishes on coral reefs. J. Exp. Mar. Biol. Ecol. 103: 21-40.

Carter, J., G.J. Marrow \& V. Pryor. 1994. Aspects of the ecology and reproduction of Nassau grouper, Epinephelus striatus, off the coast of Belize, Central America. Proc. Gulf Carib. Fish. Inst. 43: 65-111.

Cervigón, F. 1993. Los peces marinos de Venezuela. Editorial Científica Los Roques, Caracas, Venezuela.

Claro, R. 1981a. Ecología y ciclo de vida de la biajaiba Lutjanus synagris (Linnaeus) en la plataforma cubana. III. Nutrición. Cienc. Biol. 6: 93-110.

Claro, R. 1981b. Ecología y ciclo de vida del pargo criollo Lutjanus analis (Cuvier) en la plataforma cubana. Acad. Cienc. Cuba, Inf. Cient. Tec. 186: 1-83.

Claro, R. 1983. Ecology and life cycle of Lutjanus griseus (Linnaeus), from the Cuban shelf: 1. Identity, distribution and habitat, feeding and reproduction. Rep. Invest. Oceanol. Acad. Cienc. Cuba 7: 30.

Claro, R. 1994: Características generales de la ictiofauna. In R. Claro (ed.). Ecología de los peces marinos de Cuba. Inst. Oceanol. y CIQRO, Qintana Roo, México.

Cocheret, E.M., B.J.A. Pollux, I. Nagelkerken \& G. Van deer Velde. 2003. Diet shifts of Caribbean grunts (Haemulidae) and snappers (Lutjanidae) and the relation with nursery-to-coral reef Migrations. Estuar. Coast. Shelf Sci. 57: 1079-1089.

Edgar, G.J. \& C. Shaw (1995): The production and trophic ecology of shallow-water fish assemblages in southern Australia. II. Diets of fishes and trophic relationships between fishes and benthos at Western Port, Victoria. J. Exper. Mar. Biol. Ecol. 194: 83-106.

Eschmeyer, W.N. 1998. Catalog of Fishes. Spec. Pub. No.1 Centre Biod. Res. and California Acad. Sci. III. T. San Francisco, California, EEUU.

Faloh, I.G. 2001. Alimentación natural de Stegastes partitus (Poey, 1868), en el sublitoral de Ciudad de La Habana. Trabajo de Diploma, Centro de Investigaciones Marinas, Universidad de La Habana, La Habana, Cuba.

García-Arteaga, J.P., L.M. Sierra \& R. Claro. 1999. Características biológicas del bonací gato Mycteroperca tigris (Pisces: Serranidae), en la plataforma SW de Cuba. II. Alimentación, edad y crecimiento. Rev. Invest. Mar. 20: 30-36.

Guitart, J.D. 1978. Sinopsis de los peces marinos de Cuba. Científico-Técnica, Habana, Cuba.

Hernández-Hernández, I., C. Aguilar Betancourt \& G. González-Sansón. 2006. Variación estacional de la abundancia de especies de peces seleccionadas en el sublitoral rocoso de Ciudad de La Habana, Cuba. Rev. Invest. Mar. 27: 61-68.

Hixon, M.A. \& J.P. Beets. 1993. Predation, prey refuges and structure of coral reef fish assemblages. Ecol. Monogr. 6: 77-101.

Jensen, G.C. 2003. Food for Thought: Fish Diet Analysis. Fish 210: Techniques in Fisheries Fall. Lab \#9. Sin datos. $7 \mathrm{p}$.

Macpherson, E. 1981. Resource partitioning in a Mediterranean demersal fish community. Mar. Ecol. Progr. Ser. 4: 183-193.

Morato, T., E. Solá, M.P. Grós \& G. Menezes. 2003. Diets of thornback ray (Raja clavata) and tope shark (Galeorhinus galeus) in the bottom longline fishery of the Azores, northeastern Atlantic. Fish. Bull. 101: 590-602.

Pastor, L. 2000. Características biológicas de cinco especies de peces marinos ornamentales. Tesis de Maestría, Centro de Investigaciones Marinas, Universidad de La Habana, La Habana, Cuba.

Payne A., C. Smith \& A.C. Campbell. 2004. A model of oxygen-mediated filial cannibalism in fishes. Ecol. Model. 174: 253-266. 
Piet, G.J., A.B. Pfisterer \& A.D. Rijnsdorp. 1998. On factors structuring the flatfish assemblage in the southern North Sea. J. Sea Res. 40: 143-152.

Pinkas, L., M.S. Oliphant \& J.L.K. Iverson. 1971. Food habits of albacore, bluefin tuna and bonito in California waters. California. Fish and Game Fish. Bull. 152: 1-105.

Randall, J.E. 1967. Food habits of reef fishes of the West Indies. Stud. Trop. Oceanogr. 5: 665-847.

Rooker, J.R. 1995. Feeding ecology of the schoolmaster snapper Lutjanus apodus (Walbaum), from the southwestern Puerto Rico. Bull. Mar. Sci. 56: 881-894.

Sadovy, Y. \& A. Eklund. 1999. Sinopsis of biological data on the Nassau grouper, Epinephelus striatus (Bloch, 1972), and the jewfish, E. itajara (Lichtenstein, 1822). NOAA Tech. Rep. NMFS 146: 65.

Sierra, L.M. \& O.A. Popova. 1982. Feeding of Caranx ruber Bloch from the southwestern region of Cuban plataform. Rep. Invest. Oceanol. Acad. Cienc. Cuba 3: 19.

Sierra, L.M., R. Claro \& O.A. Popova. 2001. Trophic biology of the marine fishes of Cuba. In R. Claro,
K.C. Lindeman \& L.R. Parenti (eds.). Ecology of the marine fishes of Cuba. Smithsonian, Washington DC, EEUU.

Suárez, A.M, C. Aguilar \& G. González-Sansón. 1989. Comparación de dos métodos para la cuantificación del fitobentos. Rev. Invest. Mar. 10: 21-26.

Sullivan, K.M. \& M. De Garine-Wichatitsky. 1994. Energetics of juvenile Ephinephelus groupers; impact of summer temperatures and activity patterns on growth rates. Proc. Gulf Carib. Fish. Inst. 43: 148-167.

Vega-Cendejas, M.E., M. Hernández \& F. ArreguinSanchez. 1994. Trophic interrelations in a beach seine fishery from the northwestern coast of the Yucatan peninsula, Mexico. J. Fish Ecol. 44: 647-659.

\section{REFERENCIA DE INTERNET}

Salamunovich, T.J. \& R.L. Ridenhour. 1990. Food Habits of Fishes in the Redwood Creek Estuary. (Consultado: 13 octubre 2007http//www.Krisweb.com/biblio/redwood_nps_salamunovichetal_1990.pdf) 